\title{
C-17
}

\section{OPTIMIZACIÓN DEL COSTE ENERGÉTICO EN REDES DE RIEGO A PRESIÓN MEDIANTE SU REHABILITACIÓN}

\author{
Fernández García, I. $^{1}$, Rodríguez Díaz, J.A ${ }^{1}$. Montesinos, $P .{ }^{1}$, Camacho Poyato, E. ${ }^{1}$
}

\begin{abstract}
${ }^{1}$ Departamento de Agronomía. Universidad de Córdoba. Campus Rabanales, Edif. Da Vinci, 14071. Córdoba. E-mail: g52fegai@uco.es, jarodriguez@uco.es, pmontesinos@uco.es, ecamacho@uco.es
\end{abstract}

\section{Resumen}

La gestión óptima de los recursos agua y energía en todos los sectores económicos, incluida la agricultura, tiene cada vez más importancia. El sector del riego, que ha experimentado una profunda transformación hacia sistemas de riego a presión, más eficientes en el uso del agua, pero con mayor demanda energética, también requiere medidas encaminadas al aumento de la eficiencia en la gestión de estos recursos.

Este trabajo presenta una metodología fundamentada en la rehabilitación de redes de riego, mediante su rediseño, que determina el funcionamiento óptimo de la estación de bombeo que conlleva un coste de operación mínimo. La estrategia planteada se ha desarrollado en varios módulos. El primero de ellos consiste en la aplicación del algoritmo multiobjetivo NSGA-II, enlazado al simulador hidráulico EPANET, que minimiza el coste de rediseño de la red y de operación considerando un $100 \%$ de simultaneidad de hidrantes. El segundo módulo contempla un análisis de la operación a la demanda de la red, generando múltiples patrones aleatorios de hidrantes abiertos/ cerrados. El tercer módulo analiza la operación a la demanda de la red en cada uno de los rediseños propuestos en el primer módulo y determina el funcionamiento de la estación de bombeo que minimiza el coste energético, teniendo en cuenta la tarifa eléctrica.

La metodología propuesta se ha analizado en la CR EI Villar (Sevilla) proporcionando un frente de Pareto que ofrece soluciones quasi óptimas con distinto grado de satisfacción de los objetivos propuestos. Mediante el posterior análisis financiero se ha determinado que la solución más viable a largo plazo, considerando una vida útil para la red de riego de 40 años y un interés del $5 \%$, conlleva un coste de inversión asociado al reemplazo de tuberías de $205.627 €$, un coste de operación anual de $300.532 €$ y un coste total (inversión en el diseño, costes asociados a la renovación de las bombas y coste de operación) a lo largo de la vida útil de 6.019.447 €, lo que supondría un importante ahorro económico para la comunidad aplicando mayores volúmenes de agua para satisfacer las necesidades de los cultivos, en comparación con la situación actual en la que se aplica un riego deficitario debido a los elevados costes energéticos.

\section{1-Introducción}

El aumento de la eficiencia en la gestión de los recursos ha suscitado gran interés teniendo en cuenta el significativo incremento de población que se ha producido en los últimos años y la tendencia creciente esperada para las próximas décadas.

Por ello, los objetivos planteados para el año 2020 en la Estrategia Energética establecida por la Unión Europea consideran un aumento del $20 \%$ de la eficiencia energética, una reducción del $20 \%$ de las emisiones de gases de efecto invernadero y un aumento en el mismo porcentaje del uso de energías procedentes de fuentes renovables (European Commission 2010). Estos objetivos se deben considerar en todos los sectores 
económicos y el sector de la agricultura de regadío no está exento de ello. Las infraestructuras para el riego han experimentado una profunda transformación hacia sistemas a presión, más eficientes en el uso del agua pero con mayores necesidades energéticas (Corominas 2010). Este hecho, junto con el aumento de la tarifa eléctrica para el riego, han supuesto una importante reducción de los beneficios del agricultor, lo que justifica la necesidad de desarrollar estrategias que mejoren la eficiencia en el uso de la energía, uno de los retos perseguidos por la Unión Europea, y reduzcan los costes del agricultor (Rocamora et al. 2013).

Entre las medidas propuestas para minimizar el consumo de energía en las redes de riego destacan la sectorización de la red, estableciendo turnos de riego (Carrillo Cobo et al. 2011) y el control de puntos críticos, que son hidrantes con elevadas necesidades energéticas (Rodriguez Díaz et al. 2012). El análisis de estas estrategias en varias redes de riego a presión mostró ahorros energéticos que oscilaron entre el $9 \%$ y el $27 \%$ cuando se adoptó la estrategia de sectorización y entre el $5 \%$ y el $12 \%$ cuando el control de puntos críticos fue la medida analizada.

Sin embargo, las metodologías fundamentadas en el control de puntos críticos no contemplan la detección y el control de estos hidrantes de forma automática, por lo que la decisión final sobre las medidas a adoptar para mejorar el funcionamiento de los puntos críticos es del evaluador. De esta forma, una estrategia en la que ambos aspectos, detección y control de hidrantes críticos, se integren, puede contribuir a mejorar la toma de decisiones.

Para la resolución de problemas de optimización como el señalado anteriormente, se han aplicado diversos algoritmos que han permitido resolver problemas de optimización en redes de distribución de agua relativos al diseño (Farmani et al. 2007) o a la operación de la red (Fernández García et al. 2016), entre otros. Sin embargo, no se han desarrollado estrategias que combinen la optimización del diseño de la red y múltiples condiciones de funcionamiento vinculadas a la tarifa energética.

Teniendo en cuenta que una gran mayoría de las de redes de riego a presión en funcionamiento se diseñaron para operar en la condición más desfavorable y, además, muy diferentes a las actuales, en este trabajo se propone una metodología que considera, de forma conjunta, el rediseño de la red mediante la sustitución de algunas tuberías y la optimización simultánea del coste de operación bajo múltiples condiciones de carga considerando la estructura de la tarifa eléctrica.

\section{2- Metodología}

\subsection{Planteamiento del problema}

La metodología propuesta contempla la búsqueda simultánea del óptimo de dos objetivos. El primero de ellos considera la minimización del coste de rehabilitación de la red de riego mediante la determinación de los tramos de tubería que se deben reemplazar por tuberías con diámetro distinto para satisfacer las necesidades de riego, OF1. A la vez, el rediseño propuesto debe implicar que la potencia absorbida sea mínima, lo que se engloba dentro del segundo objetivo, OF2 (Fernández García et al. 2015).

Donde $j$ e $i$ son los índices relativos a la tubería y a la bomba, respectivamente, $n j$ el número de tuberías, $n_{\text {pumps }}$ el número de bombas que conforman la estación de bombeo, $U C_{j}$ el coste unitario de la tubería en función del diámetro $D_{j}\left(€ \mathrm{~m}^{-1}\right), L_{j}(\mathrm{~m})$ la longitud de la tubería $j, \gamma$ el peso específico del agua $\left(\mathrm{N} \mathrm{m}^{-3}\right), H$ la altura manométrica proporcionada por la 
estación de bombeo $(\mathrm{m}), F_{i}$ el caudal suministrado por la bomba $i\left(\mathrm{~m}^{3} \mathrm{~s}^{-1}\right)$ y $\eta_{i}$ el rendimiento de la bomba $i$.

Ambas funciones objetivo, que se determinan considerando que todos los hidrantes están abiertos, están sujetas al cumplimiento de que la presión en el hidrante más desfavorable, Pcrit, debe ser mayor o igual a la presión de servicio, Pser. Además, la resolución de OF1 y OF2, está condicionada al cumplimiento de las ecuaciones de continuidad y de conservación de la energía en redes hidráulicas.

\subsection{Rehabilitación de la red para una simultaneidad de la demanda del $100 \%$}

Para la resolución de las ecuaciones planteadas anteriormente se ha empleado como método de optimización el algoritmo multiobjetivo NSGA-II, Non-Sorted Genetic Algorithm (Deb et al. 2002), ya que se ha usado ampliamente en diversos tipos de problemas de optimización de redes de distribución de agua (e.g. Siew and Tanyimboh 2012; Carrillo Cobo et al. 2014).

El algoritmo NSGA-II comienza con la generación aleatoria de la población inicial, formada por individuos que contienen las variables del problema de optimización planteado. En este caso, cada individuo está definido por tres grupos de variables: el primer grupo se corresponde con la relación de velocidades para cada bomba de velocidad variable presente en la estación, RRS; el segundo grupo está asociado a las bombas fijas y cada variable de este grupo indica si una determinada bomba fija está funcionando o no, FP. El último grupo de variables se corresponde con los posibles diámetros para cada una de las tuberías de la red. En este caso, se optó por sustituir aquellas tuberías infradimensionadas por lo que si en el proceso de generación aleatoria de diámetros se obtenían diámetros iguales o inferiores a los actuales, se mantenían los actuales. Con la información contenida en cada individuo de la población inicial y la demanda base de los hidrantes se procedió a la evaluación de las funciones objetivo en EPANET (Rossman 2000), proporcionando el caudal suministrado por cada bomba y la presión en todos los hidrantes. A partir de la curva de rendimiento de cada bomba, se determinó éste para cada una de ellas y, por tanto, se calculó OF2. A continuación se llevaron a cabo los procesos de selección, cruzamiento y mutación para generar las sucesivas poblaciones de tal forma que los mejores individuos se mantuvieron y los peores se eliminaron. Estas últimas etapas se repitieron hasta alcanzar el número de generaciones a evaluar fijado al comienzo del proceso de optimización (Figura 1).

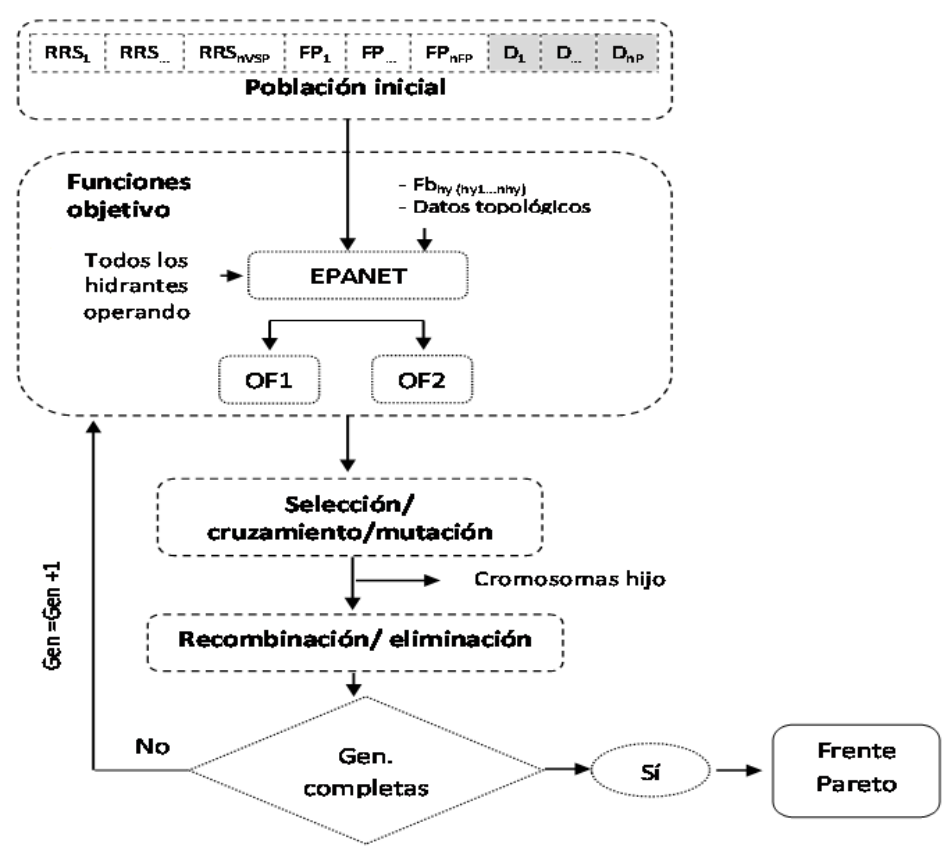

Figura 1. Esquema del proceso de optimización (epígrafe 2.2) 
El proceso de optimización se ha llevado a cabo en MATLAB (Deb et al. 2002).

\subsection{Determinación del coste de operación de los rediseños propuestos} considerando la operación a la demanda

Como resultado de la aplicación del algoritmo NSGA-II se obtiene un frente de Pareto con el conjunto de soluciones quasi-óptimas al problema planteado. Sin embargo, estas soluciones son válidas para un $100 \%$ de simultaneidad de hidrantes, lo cual no es habitual en las redes de riego. Por ello, se ha propuesto un algoritmo, compuesto por dos módulos (desarrollados en MATLAB), en el que se analiza el coste de operación, considerando la gestión a la demanda de la red, en varias soluciones seleccionadas del frente de Pareto de acuerdo al porcentaje de reducción de la potencia absorbida en relación a la inversión necesaria en la instalación (Fernández García et al. 2015). A continuación se describe el algoritmo (Figura 2).

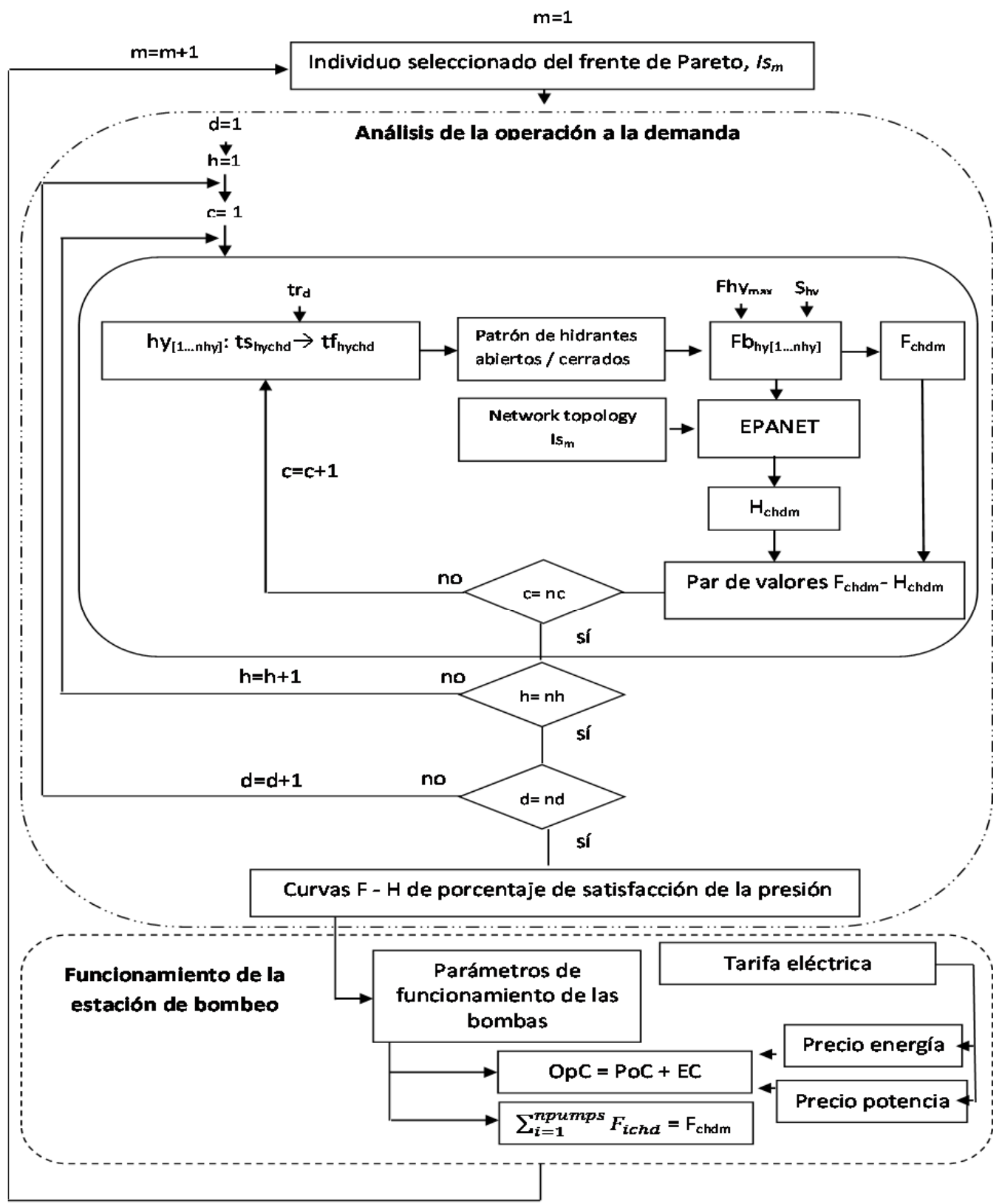

Figura 2. Esquema del análisis a la demanda de la red y la determinación del coste de operación (epígrafe 2.3) 


\subsubsection{Análisis de la operación a la demanda de la red}

En este módulo se lleva a cabo un análisis de la operación a la demanda de la red, generando múltiples patrones aleatorios de hidrantes abiertos/ cerrados. Para cada día $d$ analizado se genera un número aleatorio por hidrante hy que marca el inicio del riego, $t s_{\text {hychd. }}$. Ese número aleatorio estará incluido dentro del tiempo disponible para el riego por día. Para determinar la finalización del riego para cada hidrante, $t f_{\text {hychd, }}$ se suma el tiempo necesario de riego, $t r_{d}$, determinado a partir de las necesidades teóricas de riego del cultivo (Allen et al. 1998), al tiempo de comienzo del riego. De esta forma, para cada día se generan $c$ configuraciones en las que para cada hora $h$ se determina aleatoriamente el número de hidrantes abiertos.

En el número de horas disponibles para el riego por día influye si se establecen turnos de riego y/o si se quieren evitar las horas en las que la tarifa eléctrica es crítica en cuanto al precio. Así, cuando estos criterios se tienen en cuenta, las horas disponibles para el riego se reducen.

Una vez generados los patrones aleatorios, se lleva a cabo la evaluación de cada uno de ellos en EPANET. Cada patrón contiene información sobre los hidrantes abiertos y cerrados. Por tanto, la demanda base de cada hidrante, $F b_{h y}$, de acuerdo a cada patrón se obtiene multiplicando la superficie asociada a cada hidrante abierto, $S_{h y}$, por el caudal de

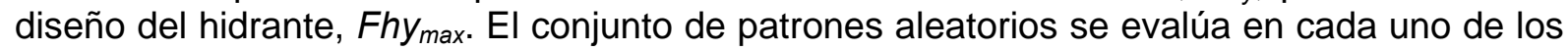
rediseños seleccionados en la etapa anterior, proporcionando el caudal y la altura manométrica que debe suministrar la estación de bombeo para garantizar la presión de servicio en todos los hidrantes, lo cual genera una nube de puntos F-H. A partir de esta nube de puntos, se pueden trazar curvas que definen, en función del caudal, el porcentaje de hidrantes que reciben la presión de servicio (Lamaddalena and Sagardoy 2000).

\subsubsection{Funcionamiento de la estación de bombeo vinculado a la tarifa eléctrica}

En el módulo anterior se obtienen las curvas del porcentaje de satisfacción de la presión en los hidrantes en función del caudal. Además, cada par de valores F-H está asociado a una hora. Para determinar el coste de operación es necesario tener en cuenta la tarifa eléctrica. La tarifa eléctrica se estructura en dos términos, un término fijo relativo a la potencia y otro variable que tiene en cuenta el consumo de energía. A su vez, las comunidades de regantes tienen que acogerse a una tarifa específica en función de la potencia contratada y de la tensión de suministro. Las comunidades de regantes suelen contratar una tarifa de alta tensión, ya sea de tres o seis periodos, en las que el precio de la potencia y la energía varían en función de cada periodo. Por tanto, el coste de operación mínimo se determinará mediante la siguiente expresión:

Donde $P o C$ es el coste asociado a la potencia, obtenido mediante la ecuación 4 y EC el coste energético, determinado mediante la ecuación 5.

$p$ es el índice para el periodo, $n p$ es el número de periodos de la tarifa eléctrica, $P_{o_{\operatorname{maxp}}}$ es la potencia contratada en función de la potencia absorbida máxima en el periodo $p$ (W) y Ppower $_{p}$ el precio del término de potencia en el periodo $p\left(€ \mathrm{~kW}^{-1}\right)$.

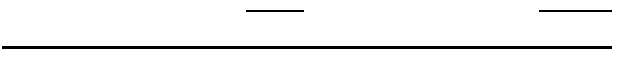


Donde $n d$ es el número de días con tarifa eléctrica diferente, $n h$ el número de horas de operación de la red de riego durante el día $d$ (24 horas si no hay turnos de riego y/o si no se evita el riego en las horas críticas), $n c$ el número de configuraciones generadas para cada hora $h$ and Penergy Pd $_{\text {el }}$ precio de la energía en función de la hora $h$ y día $d\left(€ \mathrm{kWh}^{-1}\right)$.

El algoritmo propuesto en este módulo determina el comportamiento de la estación de bombeo (relación de velocidades para las bombas de velocidad variable y el funcionamiento o no de las bombas fijas) que proporciona el par de valores F-H en función de la curva de satisfacción de la presión en los hidrantes. Para ello, se fijó el valor de $\mathrm{H}$ y se identificó la configuración de funcionamiento de la estación de bombeo que, garantizando como mínimo el valor de F, minimizaba las ecuaciones 4 y 5.

\subsection{Análisis financiero}

En este apartado se lleva a cabo un análisis financiero para seleccionar la solución más rentable a largo plazo, determinando para ello el coste total, OvC (€), que tiene en cuenta el coste de rediseño, el coste asociado al reemplazo de las bombas y el coste de operación a lo largo de la vida útil de la red:

Donde $y$ es un índice relativo al año y $k$ un índice relativo al reemplazo de las bombas. $T$ es la vida útil de la red, $s$ la vida útil de las bombas, $K$ el número de reemplazos de las bombas determinado por $T / s$ y $r$ la tasa de amortización. RC y PumpC son los costes de rehabilitación y de sustitución de las bombas, respectivamente, en $€$.

\section{$\underline{2.5 \text { Caso de estudio }}$}

La metodología propuesta se ha analizado en la CR El Villar (Sevilla), que cuenta con una superficie de $3017 \mathrm{~h}$ dedicadas a algodón, trigo, girasol y olivar. La red de riego está diseñada para aplicar un caudal máximo de $1.2 \mathrm{Ls}^{-1} \mathrm{ha}^{-1} \mathrm{a}$ una presión de servicio de 30 $\mathrm{m}$, garantizados por una estación de bombeo compuesta por 8 bombas: dos bombas de velocidad variable, con potencias de $132 \mathrm{~kW}$ y $355 \mathrm{~kW}$ y 6 fijas, de $400 \mathrm{~kW}$.

\section{3- Resultados y discusión}

\subsection{Determinación de la rehabilitación óptima de la red}

La metodología propuesta se ha aplicado a la red de riego descrita anteriormente considerando para las variables asociadas a la relación de velocidades valores comprendidos entre 0.3 y 2, valores binarios para las bombas fijas ( 0 si la bomba está parada 01 si la bomba está en funcionamiento) y los diámetros en función del catálogo seleccionado para las variables asociadas a las tuberías, los cuales variaron entre 160 y $1000 \mathrm{~mm}$, con precios unitarios comprendidos entre 11.28 y $432.57 €$.

En total, cada individuo contenía 67 variables, 2 variables para las bombas de velocidad variable, 6 variables correspondientes a las bombas fijas y 59 variables asociadas al número de tuberías que conforman la red. Para el número de individuos y para el número de generaciones se ha considerado un valor de 200 en ambos casos.

Como resultado de la aplicación del algoritmo multiobjetivo se ha obtenido un frente de Pareto que ofrece soluciones quasi óptimas con distinto grado de satisfacción de los objetivos propuestos (Figura 3). El coste de rehabilitación varió entre $44828 €$ con un valor de potencia asociada de $1973 \mathrm{~kW}$ y 3,664,885 € con una potencia asociada de $1566 \mathrm{~kW}$. Como se puede observar en la figura, el frente de Pareto desciende de forma más acusada al principio (parte izquierda de la figura), lo que indica que cuando se efectúan poco cambios en la red se obtiene una importante reducción en la potencia. Esto es debido a la presencia 
de puntos críticos que son responsables de un elevado consumo de energía (Rodriguez Díaz et al. 2012).

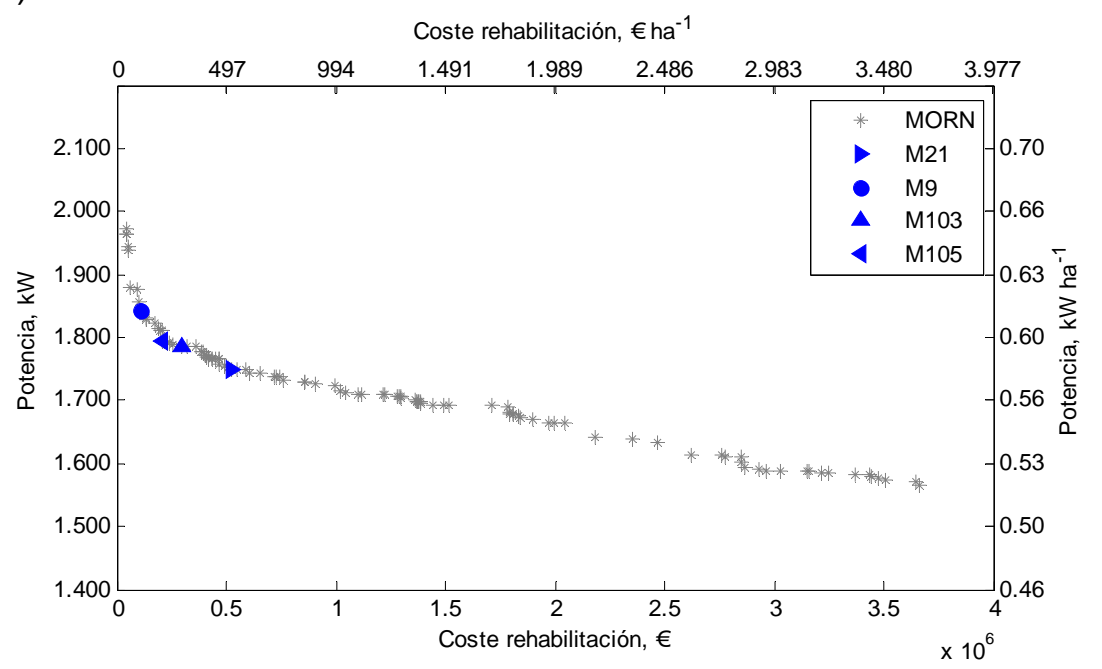

Figura 3. Frente de Pareto y soluciones seleccionadas

\subsection{Coste de operación considerando la gestión a la demanda y los nuevos} rediseños propuestos

En la etapa anterior se han obtenido rediseños quasi-óptimos de la red cuando ésta se gestiona para un $100 \%$ de simultaneidad de hidrantes. Teniendo en cuenta que el descenso más acusado en el frente de Pareto se produce en el primer cuartil (Fernández García et al. 2015), para el análisis a la demanda de la red se han seleccionado cuatro soluciones contenidas en dicho cuartil.

Diversos autores han analizado el efecto que la tarifa eléctrica tiene sobre el coste energético, determinando que concentrando el riego en las horas más baratas y, por tanto, evitando las horas críticas, se consigue una importante reducción del coste energético (Córcoles et al. 2015; Fernández García et al. 2016). Por ello, para la generación de los patrones aleatorios se evitó el riego durante las horas críticas, teniendo en cuenta para ello la estructura de la tarifa eléctrica. La tarifa eléctrica a la que se acoge esta comunidad se estructura en 6 periodos, en los que el precio varía en función de la hora del día y del día del año. La estructura y el precio de la parte regulada por el Gobierno se define en BOE 2014, mientras que la parte no regulada se ha determinado a partir del análisis del precio de la energía en el mercado libre (OMIE 2014). Por tanto, teniendo en cuenta la estructura de la tarifa eléctrica, se decidió evitar el riego entre las 10:00 y las 19:00 durante la segunda mitad de junio y durante el mes de julio (en los días laborables).

Se analizaron 30 patrones de demanda aleatorios $(n c=30)$ por hora y día diferente (nd=13) en función de la tarifa. Tras la evaluación hidráulica en EPANET, cada patrón tuvo asociado un valor de caudal y su correspondiente altura manométrica (Figura 4). En la figura 4 se observa cómo se produce una importante reducción de la altura manométrica para todo el rango de caudal en las soluciones analizadas con respecto al diseño original de la red. 
a)

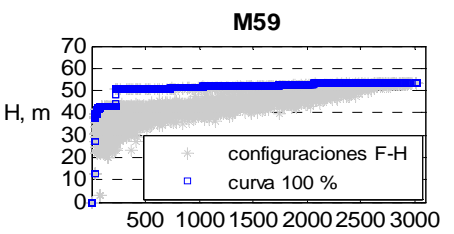

M103

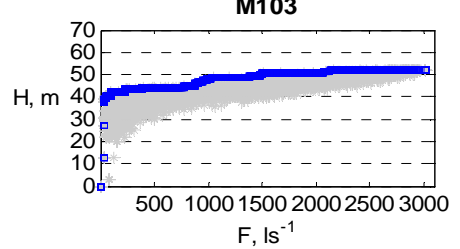

b)

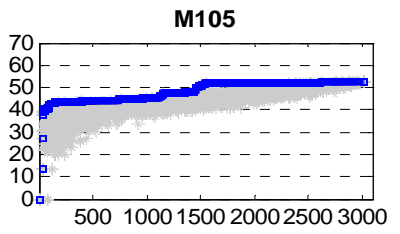

M21

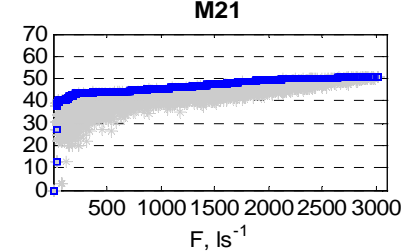

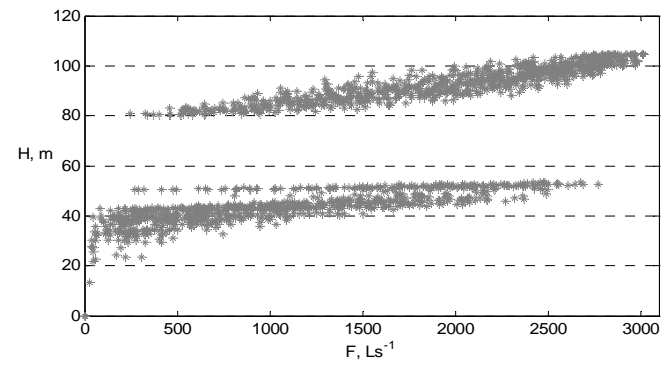

Figura 4. Pares de valores F-H en las soluciones seleccionadas (a) y en la red original (b) para cada patrón de demanda (configuración de hidrantes operativos)

Tabla 1. Número de bombas que entran en funcionamiento y costes asociados a las soluciones seleccionadas

\begin{tabular}{|c|c|c|c|c|c|c|c|c|}
\hline \multirow[t]{3}{*}{ Solución } & \multicolumn{3}{|c|}{$\begin{array}{l}\mathrm{N}^{\circ} \text { bombas en } \\
\text { funcionamiento }\end{array}$} & \multicolumn{3}{|c|}{$\begin{array}{l}\text { Coste sustitución } \\
\text { bomba, } € \text { (año } 0)\end{array}$} & \multirow[t]{2}{*}{$\begin{array}{c}\text { Coste } \\
\text { rehabilitación, } €\end{array}$} & \multirow[t]{3}{*}{$\begin{array}{c}\text { Coste } \\
\text { operación, } € \\
(\text { año 0) }\end{array}$} \\
\hline & & & & VSP1 & VSP2 & FP & & \\
\hline & VSP1 & VSP2 & $\mathrm{FP}$ & 60,264 & 109,152 & 21,152 & & \\
\hline M59 & $X^{*}$ & $x$ & 5 & & 275,176 & & 112,268 & 306,508 \\
\hline M105 & $x$ & $x$ & 5 & & 275,176 & & 205,627 & 300,532 \\
\hline M103 & $x$ & $x$ & 5 & & 275,176 & & 297,852 & 299,962 \\
\hline M21 & $x$ & $x$ & 4 & & 254,024 & & 525,484 & 295,489 \\
\hline
\end{tabular}

A partir de los rediseños seleccionados y el análisis a la demanda de la red se determinó el funcionamiento de la estación de bombeo para todo el rango de caudales. En este caso, se optó por una solución conservadora y se seleccionó la curva F-H que garantizaba la presión en todos los hidrantes (Figura 4a). En la tabla 1 se observa el número de bombas que entran en funcionamiento en la situación más desfavorable (segunda mitad de junio y julio) en cada una de las soluciones elegidas. Excepto en la solución M21, en la que en la situación más desfavorable actúan las 2 bombas de velocidad variable y 4 fijas, en las otras 3 soluciones trabajan, además de las 2 bombas de velocidad variable, 5 bombas fijas. Es importante conocer el número de bombas en funcionamiento ya que éste determina el coste total de renovación de la estación de bombeo. En este caso se ha considerado que en el año 0 se lleva a cabo la renovación de todas las bombas, lo cual implica un coste de renovación de 275,176 € para las soluciones M59, M105 y M103 y de 254,484 € para la solución M21. En cuanto al coste de rehabilitación, éste varió desde 112,268 € con un coste de operación asociado de 306,508 € hasta un coste de rehabilitación de 525,484 € con un coste de operación de 295,489 €.

\subsection{Análisis financiero}

Para el análisis financiero se ha considerado una vida útil de 40 años y un interés del $5 \%$. Teniendo en cuenta estos valores, el coste total (inversión en el diseño, costes asociados a la renovación de las bombas y coste de operación) de cada solución ha sido $6,034,617 €, 6,019,447 €, 6 ., 101,322 €$ y $6,220,335 €$ en las soluciones M59, M105, M103 y 
M21, respectivamente. Por tanto, de acuerdo a estos valores la solución más conveniente es M105, que implica el reemplazo de 10 tuberías por otras de mayor diámetro (Figura 5).

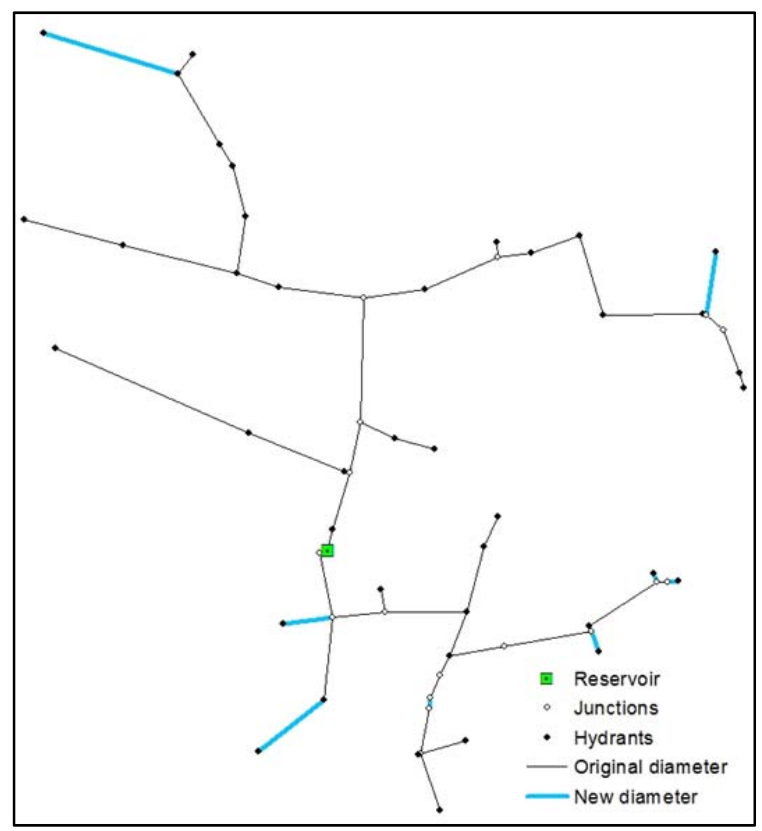

Figura 5. Esquema de la red de riego de El Villar y cambios propuestos de acuerdo a la solución M105

\section{5- Conclusiones}

La necesidad de mejorar la gestión de los recursos agua y energía repercute en todos los sectores económicos, incluida la agricultura y, más concretamente, la agricultura de regadío.

En este trabajo se ha propuesto una metodología para determinar el rediseño de la red y el funcionamiento de su estación de bombeo minimizando el coste de total (inversión y operación) teniendo en cuenta la gestión a la demanda de la red y a la estructura de la tarifa eléctrica

La metodología propuesta se ha evaluado en la CR de El Villar y tras el análisis financiero, la opción más viable, considerando una vida útil para la red de riego de 40 años y un interés del $5 \%$, conlleva un coste de inversión asociado al reemplazo de tuberías de $205.627 €$, un coste de operación anual de $300.532 €$, que supone un coste total (inversión en el diseño, costes asociados a la renovación de las bombas y coste de operación) a lo largo de la vida útil de 6.019.447 €. Aplicando el volumen total de agua requerido para satisfacer las necesidades de los cultivos tras la adopción de los cambios propuestos en la red y en el funcionamiento de la estación de bombeo, se obtendría un ahorro económico anual respecto al coste actual de operación de la red de $514.653 €(63 \%)$.

La metodología propuesta representa una estrategia fácil de implantar y adaptable en función de la gestión llevada a cabo en cada red que puede suponer un importante ahorro en el coste de operación.

\section{6- Agradecimientos}

Este trabajo se ha desarrollado en el marco del proyecto TEMAER (AGL2014-59747C2-2-R), financiado por el Ministerio de Economía y Competitividad. 


\section{7- Bibliografía}

Allen RG, Pereira L, Raes D, Smith M (1998) Crop evapotranspiration: Guidelines for computing crop water requirements. In: FAO Irrigation and Drainage Paper No. 56. Rome. Italy,

BOE (2014) Circular 3/2014, de 2 de julio, de la Comisión Nacional de los Mercados y la Competencia, por la que se establece la metodología para el cálculo de los peajes de transporte y distribución de electricidad. Boletín Of Estado 175:57158-57184.

Carrillo Cobo MT, Camacho Poyato E, Montesinos P, Rodríguez-Díaz JA (2014) New model for sustainable management of pressurized irrigation networks. Application to Bembézar MD irrigation district (Spain). Sci Total Environ 473-474:1-8. doi: 10.1016/j.scitotenv.2013.11.093

Carrillo Cobo MT, Rodríguez Díaz JA, Montesinos P, et al (2011) Low energy consumption seasonal calendar for sectoring operation in pressurized irrigation networks. Irrig Sci 29:157-169. doi: 10.1007/s00271-010-0228-2

Córcoles JI, Tarjuelo JM, Carrión PA, Moreno MÁ (2015) Methodology to Minimize Energy Costs in an On-Demand Irrigation Network Based on Arranged Opening of Hydrants. Water Resour Manag 29:3697-3710. doi: 10.1007/s11269-015-1024-9

Corominas J (2010) Agua y Energía en el riego en la época de la sostenibilidad.

Deb K, Pratap A, Agarwal S, Meyarivan T (2002) A fast and elitist multiobjective genetic algorithm: NSGA-II. IEEE Trans Evol Comput 6:182-197. doi: 10.1109/4235.996017

European Commission (2010) Energy 2020 A strategy for competitive, sustainable and secure energy. Communication from the Commission to the European Parliament, the Council, the European Economic and Social Committee and the Committee of the Regions.

Farmani R, Abadia R, Savic D (2007) Optimum Design and Management of Pressurized Branched Irrigation Networks. J Irrig Drain Eng 133:528-537. doi: 10.1061/(ASCE)0733-9437(2007)133:6(528)

Fernández García I, Creaco E, Rodríguez Díaz JA, et al (2015) Rehabilitating pressurized irrigation networks for an increased energy efficiency. Agric Water Manag. doi: 10.1016/j.agwat.2015.10.032

Fernández García I, Montesinos P, Camacho Poyato E, Rodríguez Díaz JA (2016) Energy cost optimization in pressurized irrigation networks. Irrig Sci 34:1-13. doi: 10.1007/s00271-015-0475-3

Lamaddalena N, Sagardoy JA (2000) Performance analysis of on-demand pressurized irrigation systems. In: FAO Rome. Irrigation and Drainage Paper No. 59.

OMIE (2014) OMI-Polo Español S.A. http://www.omie.es/files/flash/ResultadosMercado.swf. Accessed 1 Jan 2014

Rocamora C, Vera J, Abadía R (2013) Strategy for Efficient Energy Management to solve energy problems in modernized irrigation: Analysis of the Spanish case. Irrig Sci 31:1139-1158. doi: 10.1007/s00271-012-0394-5

Rodriguez Díaz JA, Montesinos P, Camacho Poyato E (2012) Detecting Critical Points in OnDemand Irrigation Pressurized Networks - A New Methodology. Water Resour Manag 26:1693-1713. doi: 10.1007/s11269-012-9981-8

Rossman L (2000) EPANET 2. Users manual. US Environmental Protection Agency (EPA), USA,

Siew C, Tanyimboh TT (2012) Penalty-Free Feasibility Boundary Convergent Multi-Objective Evolutionary Algorithm for the Optimization of Water Distribution Systems. Water Resour Manag 26:4485-4507. doi: 10.1007/s11269-012-0158-2 